\title{
Editorial
}

\section{Why are we doing so much cancer gene therapy? Disentangling the scientific basis from the origins of gene therapy}

The evolution of gene therapy has taken a somewhat unexpected course ... Most of the approved protocols for what is now called gene therapy involve cancer patients. This would not have been anticipated because cancer seems to be a particularly unsuitable target for the classical approach of gene replacement therapy. ${ }^{1}$

Has gene therapy really taken an unexpected course as proposed in the above citation, or is gene therapy just exploring those areas for which the science support is much stronger? I here wish to contribute to the analysis of why, given that the conceptual basis of gene therapy has now been accepted by a majority within the scientific community, ${ }^{2}$ the largest percentage of clinical trials in gene therapy are for the treatment or study of cancer (approximately $72 \%$ ), rather than any other disease; and why clinical gene therapy trials for monogenic deficiency disorders, those which initially motivated the development of gene therapy, currently only comprise approximately $16 \%$ of the total number of clinical trials.

It has been proposed that most clinical gene therapy trials are for cancer gene therapy, either because of the very high proportion of cancer patients that will die from the disease, or because its high incidence constitutes a large enough patient population for the pharmaceutical industry to direct its vast resources at diseases which can be developed into large commercial markets. As Roth and Cristiano ${ }^{1}$ argue in their recent exhaustive review on cancer gene therapy, according to the historical developments and original interpretation of gene therapy as 'replacement gene therapy', it appears somewhat difficult to understand why most gene therapy trials currently target cancer. In addition, the scientific literature has been ambivalent towards gene therapy's remit, either describing it as a therapy limited to 'gene manipulations' for the treatment of inherited diseases, or proposing that it should encompass any nucleic acid-mediated therapeutics, even if the beneficial effect of the transferred nucleic acid is unrelated to any causative genetic mutations, either known or unknown to the investigators.

The 1996 edition of Goodman and Gilman's classical pharmacology textbook ${ }^{3}$ now includes a chapter on gene therapy. This is the larger scientific field to which gene therapy belongs. In spite of its independent origin and heritage, and whether anyone would wish to rename gene therapy, it is part of pharmacology. I believe that conceiving gene therapy as the newest branch of pharma- cology will help to develop new heuristic frameworks for examining and addressing its power (effectiveness), potential (medical applications) and limitations (pharmacodynamic aspects, adverse and side-effects, toxicity).

There are some central questions that any fledgling gene therapy trial needs to address: (1) which delivery system (vector) to use; (2) when, during the time-course of the disease, do we have to treat the patient so as to achieve significant clinical benefit; (3) which organ has to be targeted; (4) which and how many of the cells within the target organ need to be transduced; (5) what level of transgene expression is needed to achieve a therapeutic effect, and does transgene expression have to be under precise regulatory controls; (6) for how long does the transgene need to be expressed to achieve a clinically relevant therapeutic effect; and (7) which is the therapeutic objective?

To answer these questions requires a comprehensive understanding of the pathophysiological basis of disease, since knowledge of the aetiology of a disease (ie genetic or environmental), and its pathophysiological mechanisms, are the pillars of new successful therapeutic approaches, like gene therapy. Furthermore, knowledge of pathophysiological mechanisms has advanced rapidly in recent years, in parallel with the progress of molecular genetics' push towards the identification of genes mutated or predisposing to human diseases. However, while genetics does pinpoint individual mutations in the genome as disease-causing mutations, it does not help us specify in which individual cell that gene's function is essential for normal cellular function. As genetics uncovers new disease-causing mutations, new tools are being developed to bridge the gap leading from gene mutation to pathophysiology. Gene therapy, just as pharmacology, thus needs to have a solid pathophysiological basis to its therapeutic implementations in order to become clinically successful.

Let us now consider the answers to the central questions when considering the implementation of a gene therapy approach to cancer: (1) which vectors? Essentially any and all gene-delivery systems developed so far have been adapted for use in cancer gene therapy. ${ }^{1}$ Retroviruses can be used to target the dividing tumour cells, but nonintegrating, high efficiency delivery systems like adenovirus vectors can also be employed, while ex vivo approaches facilitate the use of nonviral systems; ongoing clinical trials already are deploying a wide variety of different strategies; ${ }^{1}(2)$ when to treat? As soon as diagnosis is made. Presymptomatic and preventive treatment might become available in the future for patients with established high genetic risk factors; (3) which organ to 
target? The organ harbouring the tumour itself, and/or the bone marrow cells to stimulate an antitumour immune response; (4) which and how many cells to transduce? The tumour cells, if possible all, to achieve direct cell killing, or lower percentages of tumour cells if a strong bystander effect can be demonstrated, or the normal surrounding tissue, to stimulate the antitumour immune response; (5) what level and regulation of transgene expression? Enough to achieve cell killing of human tumour cells (this can easily be determined in vitro), or release differentiating and activating cytokines to stimulate the development of the immune response; (6) longevity of expression? Short term, to kill the tumour cells, or stimulate the immune response; (7) can the therapeutic objective be stated clearly? To eliminate or significantly reduce the growth of the primary tumour and its metastasis.

If we now try to apply these questions to an inherited recessive disorder, like cystic fibrosis $(\mathrm{CF})$, we will realise that answers to some questions are not yet available, and answers to others remain ambiguous: (1) genetic delivery system? Most cells to be targeted for the treatment of $\mathrm{CF}$ (eg various lung cell types) are post-mitotic nondividing cells; thus, ongoing clinical trials for CF are employing adenoviruses and liposomes; (2) when to treat? In spite of much work in this area and the obvious importance of the question, the answer to it remains unclear, with several available possibilities, ie do patients have to be treated when symptoms are first diagnosed? or do affected foetuses have to be treated in utero in order to stop the disease from developing at all? will the treatment of adults, even by gene therapy, be clinically effective, or has damage already progressed beyond repair in an affected adult? (3) which organ to target? The lungs, gastrointestinal glands or the reproductive organs? Although initial trials have targeted the upper respiratory airways, CF is a multisystemic disease, and future success will rely heavily on being able to treat a majority of symptoms throughout the body; (4) which and how many cells within the target organ to transduce? Will gene therapy have to transduce the epithelial lung cells (which are easily accessible to gene therapy vectors), or the submucous glands (which are of more restricted access)? A clear-cut response to this question remains to be provided; (5) level and regulation of transgene expression? Possibly low level of transgene expression will be enough: very elegant evidence demonstrating that a low level of transgene expression in the correct target cells will provide enough chloride channel function to be of therapeutic benefit has been presented very recently in elegant experiments using transgenic knock-out animals carrying human mutated CFTR alleles; ${ }^{4}$ (6) for how long does the transgene need to be expressed? For the whole extent of the patient's life; (7) which is the therapeutic objective? To restore lung, gastrointestinal and reproductive function. Thus, while answers to some of the central questions cannot be clearly stated and remain to be provided, other answers are clearly beyond clinical implementation at the present time.

In conclusion, cancer gene therapy is being pursued so aggressively, because available vector systems can be employed for either cell killing or immune stimulation strategies; disease pathophysiology provides clear-cut answers that can be implemented clinically, ie it is obvious when to treat the disease, which cells to target, and thus, most importantly, the therapeutic objective is unequivocal and is easy to monitor clinically. Many answers concerning gene therapy for inherited disorders and their clinical implementation still remain ambiguous, even in the cases in which clinical trials have already been ongoing (eg the various ADA deficiency trials and CF clinical trials, among others). This analysis, however, does not imply that the results of the ongoing cancer gene therapy clinical trials will necessarily have a more positive outcome than those for other diseases, but aims to indicate that further advances in our understanding of disease pathophysiology will have crucial relevance to the further development of successful gene therapy. Thus, explorations of the interplay between disease pathophysiology and gene therapy need to be exploited in order to enhance the clinical effectiveness of the future of gene therapy and its clinical trials. That cancer gene therapy can: (1) provide such clear-cut answers to the central questions of gene therapy; (2) realise a novel combinatorial (direct cytotoxicity and immune-stimulation) approach to cancer treatment founded on aetiological and pathophysiological considerations; (3) make full use of novel technology, are all substantial reasons that make cancer so attractive as a clinical target for gene therapy; notwithstanding the severity of the disease, the large numbers of patients, and the financial interests of the pharmaceutical and biotechnological industry.

\section{Acknowledgements}

I wish to acknowledge suggestions made by Maria Castro, Ricardo Dewey and Adriana Larregina, as well as ongoing discussions on gene therapy with all members of the Molecular Medicine Unit. PRL is a Research Fellow of The Lister Institute of Preventive Medicine.

PR Lowenstein
Lister Institute Professor of Molecular Medicine
and Gene Therapy
Molecular Medicine Unit
Department of Medicine
University of Manchester
Oxford Road
Manchester M13 9PT, UK

\section{References}

1 Roth JA, Cristiano RJ. Gene therapy for cancer: what have we done and where are we going? J Natl Cancer Inst 1997; 89: 21-39.

2 Friedmann T. Human gene therapy - an immature genie, but certainly out of the bottle. Nature Med 1996; 2: 144-147.

3 Eck SL, Wilson JM. Gene-based therapy. In: Limbird LE, Hardman JG (eds). Goodman and Gilman's The Pharmacological Basis of Therapeutics, 9th edn. McGraw-Hill: New York, 1996, pp 77-101.

4 Dorin JR et al. A demonstration using mouse models that successful gene therapy of cystic fibrosis requires only partial gene correction. Gene Therapy 1996; 3: 797-801. 